\title{
ON THE DECOMPOSITION OF ORTHOGONALITIES INTO SYMMETRIES
}

PETER SCHERK

1. Let $\mathfrak{F}$ be a field of characteristic $\neq 2$, and let $\Re_{n}$ denote the space of all column vectors over $\mathfrak{F}$ with $n$ components. In the following, Greek letters denote elements of $\mathfrak{F}$, while small italics $[\neq m, n, r, s]$ stand for vectors in $\Re_{n}$, and $n$-rowed squared matrices over $\mathfrak{F}$ are denoted by capital letters. A prime indicates transposition.

Let $G$ be a fixed regular symmetric matrix. Thus

$$
G=G^{\prime}, \quad|G| \neq 0 .
$$

Two vectors $a$ and $b$ are called perpendicular if $a^{\prime} G b=0$. Two subspaces $\Re^{*}$ and $\Re^{* *}$ are perpendicular if $x^{\prime} G y=0$ for all $x \subset \Re^{*}, y \subset \Re^{* *}$. Obviously, these relations are symmetric. The vectors perpendicular to a given vector respectively to a given $m$-space form an $(n-1)$ space, respectively $(n-m)$-space.

We call the matrix $T$ orthogonal if it leaves the expression $x^{\prime} G y$ unchanged for all $x$ and $y$. This condition is equivalent to

$$
T^{\prime} G T=G .
$$

If in addition

$$
\operatorname{rank}(T-I)=1 \text {, }
$$

$T$ is called a symmetry (cf. Lemma $2 ; I=$ unit matrix).

Cartan proved that every orthogonality can be decomposed into a product of $n$ or less than $n$ symmetries. A proof of his theorem can be found in Dieudonné's book. ${ }^{1}$

The purpose of this note is to show that the minimum number of symmetries into which an orthogonality $T$ can be decomposed is in general equal to

$$
m=\operatorname{rank}(T-I) .
$$

An exception occurs if and only if $G(T-I)$ is skew-symmetric. In that case, this minimum number is equal to $m+2$. For a detailed description of this case cf. the last part of this note.

2. LeMma 1. The following three sets of properties of a matrix $A$ are equivalent:

Presented to the Society, September 2, 1949; received by the editors June 14, 1949.

1 Dieudonné, Sur les groupes classiques, Actualités Scientifiques et Industrielles no. 1040, Paris, 1948, pp. 20-22. 
(a) $\operatorname{rank}(A-I)=1, A^{2}=I$;

(b) 1 is an (n-1)-fold, -1 a simple eigenvalue of $A$;

(c) There are two vectors $a$ and $b$ such that

$$
A=I+a b^{\prime}
$$

and

$$
b^{\prime} a=-2
$$

Proof. Obviously

$$
\begin{aligned}
& \operatorname{rank}(A-I)=1 \\
& \uparrow \\
& 1 \text { is an }(n-1) \text {-fold eigenvalue of } A \\
& \uparrow \\
& (2) \text { holds for suitable } a \neq 0, b \neq 0 .
\end{aligned}
$$

(a) $\rightarrow$ (b): The first part of (b) implies that $A$ has exactly one other eigenvalue $\alpha$, and this eigenvalue is simple. From $A x=\alpha x$ it follows by means of (a) that

$$
x=I x=A^{2} x=A \cdot \alpha x=\alpha \cdot A x=\alpha^{2} x .
$$

Since $x \neq 0$ and $\alpha \neq 1, \alpha=-1$.

(b) $\rightarrow$ (c): From our assumptions, there exists an $x \neq 0$ so that

$$
0=(A+I) x=\left(2 I+a b^{\prime}\right) x=2 x+b^{\prime} x \cdot a .
$$

Thus $a$ and $x$ are linearly dependent. We may choose $x=a$ and obtain $\left(2+b^{\prime} a\right) a=0$ and therefore (3).

(c) $\rightarrow(a)$ : From (3)

$A^{2}=\left(I+a b^{\prime}\right)\left(I+a b^{\prime}\right)=I+2 a b^{\prime}+a\left(b^{\prime} a\right) b^{\prime}=I+2 a b^{\prime}-2 a b^{\prime}=I$.

We call the vector $a$ isotropic if $a^{\prime} G a=0$.

Lemma 2. The following three sets of properties of a matrix $A$ are equivalent:

(a) $A$ is orthogonal, rank $(A-I)=1$;

(b) There exists a non-isotropic vector a such that

$$
A=I-\frac{2 a a^{\prime} G}{a^{\prime} G a}
$$

(c) A maps some non-isotropic vector $a$ on $-a$ and every vector perpendicular to $a$ on itself.

Proof. We first observe that (5) is equivalent to the combined three statements (2), (3), and 
$G a$ and $b$ are linearly dependent.

(a) $\rightarrow($ b) : By means of (4) we obtain (2). Thus, $A$ being orthogonal, we have

$$
0=A^{\prime} G A-G=\left(I+b a^{\prime}\right) G\left(I+a b^{\prime}\right)-G
$$

or

$$
0=b \cdot a^{\prime} G\left(I+a b^{\prime}\right)+G a \cdot b^{\prime} .
$$

This formula implies (6). Substituting $G a=\lambda b$ into (7), we obtain

$$
0=\lambda b b^{\prime}\left(I+a b^{\prime}\right)+\lambda b b^{\prime}=2 \lambda b b^{\prime}+\lambda b\left(b^{\prime} a\right) b^{\prime}
$$

hence

$$
\lambda\left(2+b^{\prime} a\right) \cdot b b^{\prime}={ }^{*} 0 .
$$

$G$ being regular, $\lambda \neq 0$. Thus (3) is also satisfied.

$(c) \rightarrow(b)$ : The assumptions (b) of Lemma 1 hold. This implies (2) and (3). From (2), $A x=x$ is equivalent to $b^{\prime} x=0$, and from our assumptions, it is also equivalent to $a^{\prime} G x=0$. Thus (6) also holds.

Obviously, (a) and (c) follow from (b).

We had defined symmetries as matrices possessing the properties (a). Thus Lemma 2 gives us two alternate definitions. From Lemma 1, we obtain the following

CoROllary. If $A$ is a symmetry, then

$$
A^{2}=I \text {. }
$$

LEMMA 3.

$$
\operatorname{rank}(A B-I) \leqq \operatorname{rank}(A-I)+\operatorname{rank}(B-I) .
$$

Proof. Put

$$
r=\operatorname{rank}(A-I), \quad s=\operatorname{rank}(B-I) .
$$

The vectors $x$ with $A x=x$, respectively $B x=x$, form an $(n-r)$-space, respectively $(n-s)$-space. If $x$ lies in the intersection of these two subspaces, then $A B x=A x=x$. Thus this intersection lies in the eigenspace of $A B$ belonging to the eigenvalue 1. The dimension of this eigenspace is therefore greater than or equal to that of this intersection. Hence it is greater than or equal to $n-r-s$. This implies (9).

If we apply (9) repeatedly to a product $T$ of $m$ symmetries, we get rank $(T-I) \leqq m$.

Thus we obtain the following 
Corollary. An orthogonality $T$ cannot be the product of less than rank $(T-I)$ symmetries.

3. LemMa 4. Let

$$
\begin{aligned}
& \operatorname{rank} S>1, \\
& S+S^{\prime} \neq 0 .
\end{aligned}
$$

Then, there exists a vector $b$ such that

$$
b^{\prime} S b \neq 0
$$

and

$$
S+S^{\prime} \neq \frac{1}{b^{\prime} S b}\left(S b \cdot b^{\prime} S+S^{\prime} b \cdot b^{\prime} S^{\prime}\right) .
$$

Proof. We have

$$
x^{\prime}\left(S+S^{\prime}\right) x=x^{\prime} S x+x^{\prime} S^{\prime} x=x^{\prime} S x+\left(x^{\prime} S x\right)^{\prime}=2 x^{\prime} S x .
$$

Thus, on account of (11), there are vectors $b$ satisfying (12). We may assume that at least one vector $b_{1}$ exists that is a solution of both (12) and

$$
S+S^{\prime}=\frac{1}{b^{\prime} S b}\left(S b \cdot b^{\prime} S+S^{\prime} b \cdot b^{\prime} S^{\prime}\right)
$$

Put

$$
c=S b_{1}, \quad d=S^{\prime} b_{1}, \quad \alpha=b_{1}^{\prime} S b_{1} .
$$

Then from (12)

$$
c \neq 0, \quad d \neq 0, \quad \alpha=b_{1}^{\prime} c=b_{1}^{\prime} d \neq 0,
$$

and from (15)

$$
S+S^{\prime}=\frac{1}{\alpha}\left(c d^{\prime}+d c^{\prime}\right) .
$$

Thus, (14) and (18) imply

$$
x^{\prime} S x=\frac{1}{2} x^{\prime}\left(S+S^{\prime}\right) x=\frac{1}{\alpha} c^{\prime} x \cdot d^{\prime} x .
$$

In particular, the quadric

$$
\mathfrak{Q} \equiv x^{\prime} S x=0
$$

is identical with the pair of [not necessarily different] $(n-1)$-spaces 
$c^{\prime} x=0$ and $d^{\prime} x=0$.

Now let $b$ be any solution of both (12) and (15). Then $S b$ and $S^{\prime} b$ are different from zero, and comparing (15) with (18), we see that either $S b$ is a multiple of $c$ and $S^{\prime} b$ is one of $d$, or $S b$ is a multiple of $d$, while $S^{\prime} b$ is one of $c$.

We first consider the case that a solution $b_{2}$ of (12) and (15) exists such that $S b_{2}$ is not a multiple of $c$. Then $S b_{2}$ is a multiple of $d$, and the vectors $c$ and $d$ are linearly independent. Replacing $b_{2}$ by a suitable multiple, we may assume

$$
S b_{2}=d, \quad S^{\prime} b_{2}=\beta c, \quad \beta \neq 0 .
$$

Substituting $b=b_{2}$ into (15), we obtain

$$
S+S^{\prime}=\frac{\beta}{b_{2}^{\prime} S b_{2}}\left(d c^{\prime}+c d^{\prime}\right),
$$

and therefore, on account of (18),

$$
b_{2}^{\prime} S b_{2}=\alpha \beta \text {. }
$$

Thus (19) implies

$$
b_{2}^{\prime} d=\alpha \beta, \quad b_{2}^{\prime} c=\alpha .
$$

The vectors

$$
S\left(b_{1} \pm b_{2}\right)=c \pm d
$$

are multiples neither of $c$ nor of $d$ [cf. (16) and (19)]. Hence the two vectors $b_{1} \pm b_{2}$ cannot solve the system (12), (15). Now, from (16), (17), (19), (20)

$$
\begin{aligned}
& \left(b_{1}+b_{2}\right)^{\prime} S\left(b_{1}+b_{2}\right)-\left(b_{1}-b_{2}\right)^{\prime} S\left(b_{1}-b_{2}\right) \\
& \quad=2\left(b_{1}^{\prime} S b_{2}+b_{2}^{\prime} S b_{1}\right)=2\left(b_{1}^{\prime} d+b_{2}^{\prime} c\right)=2(\alpha+\alpha)=4 \alpha \neq 0 .
\end{aligned}
$$

Hence, at least one of the two vectors $b_{1} \pm b_{2}$ satisfies (12). As it cannot satisfy (15), it is a solution of both (12) and (13).

Suppose now that every solution $b$ of (12) and (15) is mapped by $S$ on a multiple of $c$. The set of all vectors $x$ for which $S x$ is a multiple of $c$ form a subspace $\Re_{c}$ of $\Re_{n}$. From (10), $\Re_{c}$ is a proper subspace of $\Re_{n}$. Hence its dimension is not greater than $n-1$. It suffices to show that there are vectors in $\Re_{n}$ that belong neither to $\Re_{c}$ nor to the quadric $\mathfrak{Q}$.

We had $b_{1} \subset \Re_{c}-\mathfrak{Q}$. Since $\mathfrak{Q}$ was a pair of $(n-1)$-spaces, there exists a vector $b_{2} \subset \mathfrak{Q}-\Re_{c}$. Thus, the straight line

$$
b=(1-\lambda) b_{1}+\lambda b_{2}
$$


lies neither in $\Re_{c}$ nor in $\mathfrak{Q}$. Hence it has exactly one point in common with $\Re_{c}$ and at most two points with the pair $\mathfrak{Q}$ of $(n-1)$-spaces. Altogether, the straight line (21) meets the union $\Re_{c}+\mathfrak{Q}$ in not more than three points. If $\mathfrak{F}$ is not the prime field $\mathfrak{F}_{3}$ of three elements, this line contains more than three points. In particular, it contains points outside of $\Re_{c}+\mathfrak{Q}$.

If $\mathfrak{F}=\mathfrak{F}_{3}$, then an $(n-1)$-space contains $3^{n-1}$ points. Since $\Re_{c}$ has a dimension not greater than $n-1$ and since $\Re_{c}$ and $\mathscr{Q}$ have the origin in common, the set $\Re_{c}+\mathscr{Q}$ contains less than $3.3^{n-1}$ points, thus fewer points than the whole $\Re_{n}$. Hence, there are points outside of $\Re_{c}+\mathfrak{Q} .^{2}$

Lemma 5. Suppose $T$ is orthogonal, $G(T-I)$ is not skew-symmetric, and

$$
m=\operatorname{rank}(T-I)>1 .
$$

Then there exists an orthogonality $U$ such that

(a) $T$ is the product of $U$ by a symmetry,

(b) $\operatorname{rank}(U-I)=m-1$,

(c) $G(U-I)$ is not skew-symmetric.

Proof. Put

$$
T_{0}=T-I, \quad S=G T_{0} .
$$

We rewrite the orthogonality definition (1) in terms of $T_{0}$ and $S$ :

$$
T^{\prime} G T-G=\left(T_{0}+I\right)^{\prime} G\left(T_{0}+I\right)-G=0
$$

or

$$
T_{0}^{\prime} G T_{0}+S+S^{\prime}=0 .
$$

$G$ being regular, we have

$$
\operatorname{rank} S=\operatorname{rank} T_{0}=m>1 .
$$

Thus $S$ satisfies the assumptions of Lemma 4 , and there is a vector $b$ such that

$$
b^{\prime} S b \neq 0
$$

and

2 The proof of Lemma 4 can be simplified considerably if the case $\mathfrak{F}=\mathfrak{F}_{3}$ is excluded. If $\mathfrak{F}$ is the real field, this lemma is trivial. The matrix $S b \cdot b^{\prime} S+S^{\prime} b \cdot b^{\prime} S$, $-b^{\prime} S b\left(S+S^{\prime}\right)$ depends continuously on $b$. If it vanishes outside of the quadric $\mathfrak{Q}$ identically, then it will also vanish on $\mathfrak{Q}$. Hence $S b \cdot b^{\prime} S+S^{\prime} b \cdot b^{\prime} S^{\prime}=0$ for all $b \subset \mathbb{Q}$. Therefore, $\mathfrak{Q}$ would be contained in the pair of at most $(n-2)$-dimensional subspaces $S b=0$ and $S^{\prime} b=0$, which is impossible. 


$$
S+S^{\prime} \neq \frac{1}{b^{\prime} S b}\left(S b \cdot b^{\prime} S+S^{\prime} b \cdot b^{\prime} S^{\prime}\right)
$$

Let $\Re_{n-m}$ be the eigenspace belonging to the eigenvalue 1 of $T$. From the regularity of $G$ and of $T=T_{0}+I$

$$
x \subset \Re_{n-m} \leftrightarrow T_{0} x=0 \leftrightarrow S x=0 \leftrightarrow S^{\prime} x=0 \quad \text { [cf. (22)]. }
$$

In particular, from (23),

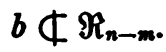

Define

$$
a=T_{0} b .
$$

Then, from (25),

$$
a^{\prime} G x=b^{\prime} S^{\prime} x=0 \quad \text { for all } x \subset \Re_{n-m} .
$$

Furthermore, (27), (22), and (14) yield

$$
a^{\prime} G a=b^{\prime} T_{0}^{\prime} G T_{0} b=-b^{\prime}\left(S+S^{\prime}\right) b=-2 b^{\prime} S b .
$$

Thus, from (27) and (23)

$$
a^{\prime} G a=-2 b^{\prime} S b=-2 b^{\prime} G a \neq 0 .
$$

We now put

$$
A=I-\frac{2 a a^{\prime} G}{a^{\prime} G a}, \quad U=A T .
$$

Thus, $A$ is a symmetrv [cf. Lemma 2]; and with $A$ and $T, U$ is an orthogonality. From (8)

$$
T=A^{2} T=A U .
$$

We now verify that $U$ also has the required properties (b) and (c). If $x \subset \Re_{n-m}$, then on account of (28)

$$
U x=A T x=A x=x-\frac{2 a^{\prime} G x}{a^{\prime} G a} \cdot a=x .
$$

Furthermore, we obtain from (30), (27), and (29)

$$
(T-A) b=\left(T_{0}+\frac{2 a a^{\prime} G}{a^{\prime} G a}\right) b=a+\frac{2 a^{\prime} G b}{a^{\prime} G a} a=a-a=0,
$$

hence

$$
U b=A T b=A^{2} b=b .
$$


Thus the eigenspace of $U$ belonging to the eigenvalue 1 contains both $\Re_{n-m}$ and $b$. As $b \nsubseteq \Re_{n-m}$ [cf. (26)], its dimension is not less than $n-m+1$, that is,

$$
\operatorname{rank}(U-I) \leqq m-1 .
$$

Thus our assertion (b) follows by applying Lemma 3 to $T=A U$.

It remains to be shown that $G(U-I)$ is not skew-symmetric. We have

$$
\begin{aligned}
U-T & =A T-I=\left(I-\frac{2 a a^{\prime} G}{a^{\prime} G a}\right)\left(T_{0}+I\right)-I \\
& =T_{0}-2 \frac{T_{0} b b^{\prime} T_{0}^{\prime} G\left(T_{0}+I\right)}{-2 b^{\prime} S b}=T_{0}-\frac{T_{0} b b^{\prime} S}{b^{\prime} S b}
\end{aligned}
$$

[cf. (27), (29), and (22)]. Thus

$$
G(U-I)=S-\frac{S b b^{\prime} S}{b^{\prime} S b},
$$

and (c) follows from (24).

Theorem 1. Suppose $T$ is orthogonal and $G(T-I)$ is not skew-symmetric. Then $T$ can be written as a product of $m=\operatorname{rank}(T-I)$ symmetries, but not of less than $m$.

Proof. For $m=0$ and $m=1$ our statement is trivial [cf. Lemma 2]. Suppose it is proved up to $m-1 \geqq 1$. From the corollary of Lemma 3, $T$ cannot be a product of fewer than $m$ symmetries. Thus, our theorem follows from Lemma 5 and our induction assumption.

4. From now on we assume not only that $T$ is orthogonal but also that $G(T-I)$ is skew-symmetric. Put $T_{0}=T-I, m=\operatorname{rank} T_{0}$. Being the rank of the skew-symmetric matrix $G T_{0}, m$ is even. As the case $m=0$ is trivial, we may assume $m \geqq 2$.

From our assumptions

$$
G T_{0}+T_{0}^{\prime} G=0 .
$$

Hence

$$
T^{\prime} G T-G=\left(T_{0}^{\prime}+I\right) G\left(T_{0}+I\right)-G=T_{0}^{\prime} G T_{0}+G T_{0}+T_{0}^{\prime} G=0
$$
implies

$$
T_{0}^{\prime} G T_{0}=0 .
$$

We obtain from (31) and (32) $G T_{0}{ }^{2}+T_{0}{ }^{\prime} G T_{0}=G T_{0}^{2}=0$. Since $G$ is 
regular, it follows that

$$
T_{0}^{2}=0 .
$$

Our definition of $m$ implies that $T_{0}$ maps the whole space $\Re_{n}$ on an $m$-space $\Re_{m}$, and the set of the vectors $x$ with $T_{0} x=0$ forms an $(n-m)$-space $\Re_{n-m}$. From (33)

$$
\Re_{m} \subset \Re_{n-m}
$$

therefore

$$
m \leqq n-m \text { or } m \leqq n / 2 \text {. }
$$

If $x \subset \Re_{m}, y \subset \Re_{n-m}$, then $x=T_{0} z$ for some $z$, and $T_{0} y=0$. From (31)

$$
x^{\prime} G y=z^{\prime} T_{0}^{\prime} G y=-z^{\prime} G T_{0} y=0 .
$$

So, the two spaces $\Re_{m}$ and $\Re_{n-m}$ are perpendicular. Since the vectors perpendicular to $\Re_{n-m}$ form an $m$-space containing $\Re_{m}$, this $m$-space is equal to $\Re_{m}$. Hence, a vector is perpendicular to $\Re_{n-m}$ if and only if it lies in $\Re_{m}$. We obtain from (34) that $\Re_{m}$ is perpendicular to itself. In particular, every vector in $\Re_{m}$ is isotropic.

Let

$$
A=I-\frac{2 a a^{\prime} G}{a^{\prime} G a}
$$

be an arbitrary symmetry. Thus $a$ may be any non-isotropic vector. Since it cannot lie in $\Re_{m}$, it is not perpendicular to $\Re_{n-m}$, and the $(n-1)$-space $\Re_{n-1}$ perpendicular to $a$ does not contain $\Re_{n-m}$. The intersection

$$
\Re_{n-m-1}=\Re_{n-1} \cdot \Re_{n-m}
$$

of these two spaces is therefore an $(n-m-1)$-space.

Let $U=A T$. We first show that $\Re_{n-m-1}$ is the eigenspace of $U$ belonging to the eigenvalue 1 . This implies in particular that

$$
\operatorname{rank}(U-I)=m+1 \text {. }
$$

If $x \subset \Re_{n-m-1}$, then from (36) and (35) $U x=A T x=A x=x$.

Conversely, suppose $U x=x$. Then $T x=A^{2} T x=A U x=A x$ and hence

$$
T_{0} x=(T-I) x=(A-I) x=-\frac{2 a^{\prime} G x}{a^{\prime} G a} \cdot a,
$$


From (38) and (32)

$$
x^{\prime} T_{0}^{\prime} G T_{0} x=\left(\frac{2 a^{\prime} G x}{a^{\prime} G a}\right)^{2} a^{\prime} G a=0 .
$$

Since $a^{\prime} G a \neq 0$, this implies $a^{\prime} G x=0$ or $x \subset \Re_{n-1}$. Going back to (38), we obtain $T_{0} x=0$ or $x \subset \Re_{n-m}$. Thus $x \subset \Re_{n-m-1}$. This proves the above statement.

Since $m$ is even,

$$
\operatorname{rank} G(U-I)=\operatorname{rank}(U-I)=m+1
$$

is odd. Hence, $G(U-I)$ cannot be skew-symmetric.

From Theorem $1, U$ is a product of $m+1$ symmetries. Hence $T=A U$ can be written as a product of $m+2$ symmetries. Suppose we have expressed $T$ as a product of $k$ symmetries. Then we may put $T=A U$ where $A$ is a symmetry (35) and $U$ is the product of $k-1$ symmetries. Since $U=A T$, we arrive again at (37). From the corollary to Lemma 3,

$$
k-1 \geqq m+1, \quad \text { that is, } k \geqq m+2 .
$$

So we have the following theorem.

Theorem 2. Suppose $T$ is orthogonal and $G(T-I)$ is skew-symmetric. Let

$$
m=\operatorname{rank}(T-I) .
$$

Then

$$
m \equiv 0(\bmod 2) \quad \text { and } m \leqq n / 2,
$$

and $T$ can be decomposed into a product of $m+2$ but not fewer than $m+2$ symmetries.

In order to find these transformations $T \neq 1$, we choose a basis whose $m>0$ first vectors span $\Re_{m}$. If $m<n / 2$, the next $n-2 m$ vectors of this basis shall lie in $\Re_{n-m}$. Since

$$
x^{\prime} G y=y^{\prime} G x=0 \quad \text { for all } x \subset \Re_{m}, y \subset \Re_{n-m},
$$

$G$ has in these coordinates the form

$$
G=\left(\begin{array}{ccc}
0 & 0 & G_{1}^{\prime} \\
0 & G_{2} & * \\
G_{1} & * & *
\end{array}\right) \text { if } m<\frac{n}{2}, G=\left(\begin{array}{cc}
0 & G_{1}^{\prime} \\
G_{1} & *
\end{array}\right) \text { if } m=\frac{n}{2} .
$$

Here $G_{1}$ and $G_{2}$ are regular square matrices; $G_{1}$ is $m$-rowed, $G_{2}$ is 
$(n-2 m)$-rowed and symmetric. We have

$T_{0} x \subset \Re_{m}$ for all $x \subset \Re_{n}$ and $T_{0} y=0$ for all $y \subset \Re_{n-m}$.

Hence

$$
T_{0}=\left(\begin{array}{cc}
0 & T_{1} \\
0 & 0
\end{array}\right)
$$

where $T_{1}$ is an $m$-rowed squared matrix. Its regularity follows from rank $T_{0}=m$. We obtain

$$
G T_{0}=\left(\begin{array}{cc}
0 & 0 \\
0 & G_{1} T_{1}
\end{array}\right)
$$

Thus (32) and (33) are satisfied. Finally, $G T_{0}$ is skew-symmetric if and only if the same holds true of $G_{1} T_{1}$. This leads to the following construction:

Choose $n \geqq 4$ arbitrarily, $m>0$ according to (39) and then $G$ according to $(40)$. Then

$$
T_{0}=\left(\begin{array}{cc}
0 & G_{1}^{-1} T_{2} \\
0 & 0
\end{array}\right)
$$

where $T_{2}$ may be any $m$-rowed regular skew-symmetric matrix.

If we take, for example, $n=4, m=2$,

$$
G_{1}=\left(\begin{array}{ll}
1 & 0 \\
0 & 1
\end{array}\right) \text { and } T_{2}=\left(\begin{array}{rr}
0 & 1 \\
-1 & 0
\end{array}\right)
$$

we may put

$$
G=\left(\begin{array}{llll}
0 & 0 & 1 & 0 \\
0 & 0 & 0 & 1 \\
1 & 0 & 0 & 0 \\
0 & 1 & 0 & 0
\end{array}\right) \text { and } T_{0}=\left(\begin{array}{rrrr}
0 & 0 & 0 & 1 \\
0 & 0 & -1 & 0 \\
0 & 0 & 0 & 0 \\
0 & 0 & 0 & 0
\end{array}\right)^{8}
$$

\section{UNIVERSITY OF SASKaTChewaN}

Professor Coxeter has made the following comment on the last example: "A space with two space-like and two time-like dimensions admits a transformation leaving a whole plane invariant although it is not merely a rotation. The explanation is, of course, that this invariant plane is isotropic." 